\title{
Physiology Principles Underlying Goal Directed Therapies in Children
}

\section{Kumba C*}

Department of Pediatric Anesthesia and Critical Care, Necker Enfants Malades University Hospital, Assistance Publique Hôpitaux de Paris, APHP, University of Paris, Paris, France

Ecole Doctorale 563 Médicament-Toxicologie-Chimie-Imageries (MTCI), Université de Paris, Paris, France

ISSN: 2576-9200

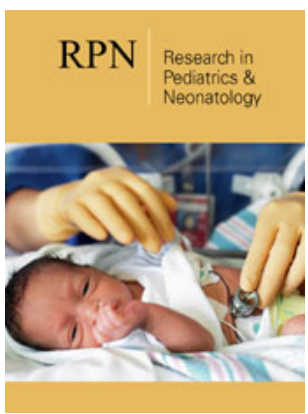

*Corresponding author: Kumba C, Department of Pediatric Anesthesia and Critical Care, Necker Enfants Malades University Hospital, Assistance Publique Hôpitaux de Paris, APHP, University of Paris, Paris, France

Ecole Doctorale 563 MédicamentToxicologie-Chimie-Imageries (MTCI), Université de Paris, Paris, France

Submission: 侮 May 15, 2020

Published: 制June 04, 2020

Volume 4 - Issue 4

How to cite this article: Kumba C. Physiology Principles Underlying Goal Directed Therapies in Children. Res Pediatr Neonatol. 4(4). RPN. 000591. 2020. DOI: $10.31031 /$ RPN.2020.04.000591

Copyright $\left(C^{\circ}\right.$ Kumba C. This article is distributed under the terms of the Creative Commons Attribution 4.0 International License, which permits unrestricted use and redistribution provided that the original author and source are credited.

\begin{abstract}
Background: Goal directed therapies (GDT) include goal directed fluid and hemodynamic therapy (GDFHT), transfusion goal directed protocols (TGDP) and enhanced recovery after surgery (ERAS). These GDT share common aims which are to optimize tissular oxygen delivery $\left(\mathrm{DO}_{2}\right)$, oxygen consumption $\left(\mathrm{VO}_{2}\right)$ and extraction ratio $\left(\mathrm{O}_{2} \mathrm{ER}\right)$.
\end{abstract}

Objectives: This editorial on the Thesis Project entitled 'Do goal directed therapies improve postoperative outcome in children' highlights the physiology and rationale of GDT.

Methods: Editorial on the rationale of the Thesis Project in GDT in children.

Result: GDFHT, TGDP and ERAS have the same aim which is the optimization of tissular $\mathrm{DO}_{2}, \mathrm{VO}_{2}$ and $\mathrm{O}_{2} \mathrm{ER}$ to avoid and prevent organ dysfunction.

Conclusion: Understanding the physiology of GDT is important for optimal patients management.

Keywords: Goal directed fluid and hemodynamic therapy; Transfusion goal directed protocols; Enhanced recovery after surgery; Postoperative outcomes, Children; Oxygen delivery; Oxygen consumption; Oxygen extraction ratio; Tissular perfusion pressure

\section{Introduction}

A Thesis Project has been undertaken which has the objectives to determine the impact of Goal directed therapies on postoperative outcome in children [1]. The background of this Thesis Project were the results of five retrospective observational studies realized in the pediatric surgical settings [2-6]. These studies had the objectives of determining predictors of adverse postoperative outcomes in the surgical pediatric population. The aim of this Thesis Project is to bring improvement measures in domains where predictors of postoperative adverse outcomes were identified. In order to implement these measures prospective and randomized controlled trials need to be developed. The hypothesis of the Thesis is by implementing goal directed therapies in fields were predictors of pejorative postoperative outcome have been identified, outcome in terms of postoperative morbidity and length of hospital stay will be improved.

\section{Rationale of Goal Directed Therapies}

Goal directed therapies (GDT) include goal directed fluid and hemodynamic therapy (GDFHT), transfusion goal directed protocols (TGDP) and enhanced recovery after surgery (ERAS) [7-33]. GDFHT, TGDP and ERAS share common goals. These aims are to optimize oxygen delivery $\left(\mathrm{DO}_{2}\right)$, oxygen consumption $\left(\mathrm{VO}_{2}\right)$ and oxygen extraction in different tissues of the organism. Considering the following equations, one will understand the physiology and the basis of GDT $[14,22,23,34,36]$.

$$
\begin{aligned}
& \mathrm{DO}_{2}=\mathrm{COxCaO}_{2}=\mathrm{COx}\left(\mathrm{Hbx} 1.31 \mathrm{xSaO}_{2}+0.0031 \times \mathrm{PaO}_{2}\right) \\
& \mathrm{VO}_{2}=\mathrm{CO}\left(\mathrm{CaO}_{2}-\mathrm{CvO}_{2}\right) \\
& \mathrm{CaO}_{2}=\mathrm{Hbx}_{1.31 \times \mathrm{SaO}_{2}}+0.0031 \mathrm{xPaO}_{2} \\
& \mathrm{CvO}_{2}=\mathrm{Hbx} 1.31 \mathrm{xSvO}_{2}+0.0031 \mathrm{xvO}_{2} \\
& \mathrm{O}_{2} \mathrm{ER}=\mathrm{CaO}_{2}-\mathrm{CvO}_{2} / \mathrm{CaO}_{2}=\mathrm{SaO}_{2}-\mathrm{SvO}_{2} / \mathrm{SaO}_{2}=\mathrm{VO}_{2} / \mathrm{DO}_{2}
\end{aligned}
$$




\section{$\mathrm{CO}=\mathrm{SV} \times \mathrm{HR}=\mathrm{VTIXD}^{2} \mathrm{x} \Pi / 4 \mathrm{xHR}$}

$\mathrm{SV}=$ Aortic Velocity Time Integral $\mathrm{x}$ area of the aortic valve $=\mathrm{VTIXD}^{2} \mathrm{x} \Pi / 4$

$\mathrm{PP}=\mathrm{SVR} \times \mathrm{CO}$

Where $\mathrm{CO}=$ Cardiac output, $\mathrm{SV}=$ Stroke volume, $\mathrm{HR}=$ Heart rate, $\mathrm{PP}=$ Tissular perfusion pressure, $\mathrm{SVR}=$ Systemic vascular resistance, $\mathrm{VTI}=$ Aortic velocity time integral, $\mathrm{D}=$ Diameter of the aortic valve, $\mathrm{CaO}_{2}=$ Arterial oxygen content, $\mathrm{CvO}_{2}=$ Venous oxygen content, $\mathrm{Hb}=$ Hemoglobin levels, $\mathrm{PaO}_{2}=$ Arterial oxygen partial pressure, $\mathrm{PvO}_{2}=$ Venous oxygen partial pressure, $\mathrm{SaO}_{2}=$ Arterial oxygen saturation, $\mathrm{SvO}_{2}=$ Venous oxygen saturation, $\mathrm{O}_{2} \mathrm{ER}=$ Oxygen extraction ratio.

The determinants of $\mathrm{DO}_{2}$ are $\mathrm{CO}, \mathrm{Hb}, \mathrm{SaO}_{2}, \mathrm{PaO}_{2}$.

The determinants of $\mathrm{VO}_{2}$ are $\mathrm{CO}, \mathrm{Hb}, \mathrm{SaO}_{2}, \mathrm{PaO}_{2}, \mathrm{SvO}_{2}, \mathrm{PvO}_{2}$.

The determinants of $\mathrm{CO}$ are SV and heart rate.

The determinants of SV are afterload [ventricular relaxation and compliance (diastolic function); systemic arterial blood pressure, systemic vascular resistance; pulmonary arterial pressure, pulmonary vascular resistance], preload (volemia) and heart contractility (systolic function). The determinants of tissular perfusion pressure are SVR and CO.

Optimizing $\mathrm{DO}_{2}, \mathrm{VO}_{2}$ and $\mathrm{O}_{2}$ ER means that the demand $\left(\mathrm{VO}_{2}\right)$ has to be fulfilled by the offer $\left(\mathrm{DO}_{2}\right)$ [23]. If $\mathrm{VO}_{2}$ exceeds $\mathrm{DO}_{2}$, the tissues have to increase oxygen extraction in order to fulfill the demand [23]. If $\mathrm{VO}_{2}$ exceeds $\mathrm{DO}_{2}$ and oxygen extraction does not increase, a deficit in oxygen will occur which will lead to anaerobic metabolism which will increase lactate production and decrease tissular perfusion which will lead to organ dysfunction [22,23,3436]. In normal states, $\mathrm{VO}_{2}$ is independent of $\mathrm{DO}_{2}$. If $\mathrm{DO}_{2}$ decreases to a critical state, $\mathrm{VO}_{2}$ becomes dependent on oxygen delivery. In this situation $\mathrm{O}_{2}$ ER increases to fulfill $\mathrm{VO}_{2}$. However $\mathrm{O}_{2}$ ER cannot increase continuously when $\mathrm{DO}_{2}$ decreases under the critical point. In this state of $\mathrm{DO}_{2}$ dependency, hypoxia occurs and leads to organ dysfunction and lactate levels increase due to anaerobic metabolism [22,23,34-36].

Considering the goal directed fluid and hemodynamic therapy (GDFHT) point of view [9-20]. The objectives of the GDFHT are to optimize $\mathrm{DO}_{2}$ to the tissues and tissular $\mathrm{VO}_{2}$. $\mathrm{DO}_{2}$ can be optimized in GDFHT by increasing CO. CO can be increased by optimizing SV. SV can be assessed echocardiographically with aortic peak velocity variation $(\Delta$ Vpeak), aortic velocity time integral (VTI) and distance minute (DM) at the aortic valve [9-20]. Assessing aortic velocity time integral (VTI) and aortic peak velocity variation $(\Delta$ Vpeak) will determine fluid responsiveness if fluid therapy with crystalloids and or colloids is necessary or vasopressor-inotropic therapy to increase SV and thus cardiac output [14]. As precised here above the determinants of tissular perfusion are systemic vascular resistance and cardiac output. Tissular perfusion pressure can decrease if SVR is low and or if $\mathrm{CO}$ is low. Optimizing SV with fluid and or inotropic therapy and SVR with vasopressor therapy will increase tissular perfusion pressure. In GDFHT, $\mathrm{DO}_{2}$ can be optimized by increasing $\mathrm{CO}$ as explained here above. GDFHT aims to avoid $\mathrm{DO}_{2}$ dependency states and prevent organ dysfunction [22,23,34-36].

Considering the transfusion goal directed protocols (TGDP) point of view [23-28,33,36]. Optimizing $\mathrm{DO}_{2}$ and $\mathrm{VO}_{2}$ will be achieved by optimizing hemoglobin levels. The ideal hemoglobin level is one that avoids situations where $\mathrm{VO}_{2}$ is dependent on $\mathrm{DO}_{2}$ and depends on the clinical context [22]. Since transfusion of all sorts of blood products has been related to postoperative morbidity in terms of organ dysfunction among others, it is important to transfuse the right product at the right time [2-5]. Using point of care viscoelastic methods can be helpful to guide and transfuse correctly [24-28]. These point of care devices have been shown to reduce length of hospital stay in hemorrhagic surgeries in children [28]. Maintaining the optimal hemoglobin levels to avoid situations where $\mathrm{VO}_{2}$ exceeds $\mathrm{DO}_{2}$ is mandatary since anemia has been related to increased mortality in children [26].

The important issue is to avoid unnecessary blood product administration which increases morbidity [2-5] and also avoid unnecessary anemia which can increase mortality [26]. In hemorrhagic settings like surgery, point of care devices can be useful to detect coagulation disorders which can be promptly treated with the appropriate blood products. Correcting coagulation disorders will further reduce bleeding which will avoid hemoglobin level decrease and thus avoid red blood cell transfusion. The use of TGDP is beneficial in bleeding situations to guide the correct use of blood products. Transfusion of red blood blood cells has been shown to reduce oxygen extraction ratio in cardiac surgical children with high extraction ratio [33]. The ideal hemoglobin level is the one that avoids to reach the oxygen delivery dependency state and depends on patients clinical context. The objectives of TGDP are to avoid and prevent situations where $\mathrm{VO}_{2}$ becomes dependent on $\mathrm{DO}_{2}$. $\mathrm{DO}_{2}$ dependency state can lead to organ dysfunction (Table 1).

Table 1: Factors influencing determinants of $\mathrm{DO}_{2}$ and $\mathrm{VO}_{2}$.

\begin{tabular}{|c|c|c|}
\hline Determinants of $\mathbf{D O}_{2}$ & Determinants of $\mathbf{V O}_{2}$ & Influencing Factors \\
\hline $\mathrm{CO}$ & $\mathrm{CO}$ & Goal directed Fluid and hemodynamic therapy: Fluid therapy and or vasopressor-inotropic therapy \\
\hline $\mathrm{Hb}$ & $\mathrm{Hb}$ & Transfusion Goal Directed Protocols \\
\hline $\mathrm{SaO}_{2}$ & $\mathrm{SaO}_{2}$ & ERAS: Protective ventilation, optimal oxygen therapy, physiotherapy, optimal pain therapy \\
\hline $\mathrm{PaO}_{2}$ & $\mathrm{PaO}_{2}$ & ERAS: Protective ventilation, optimal oxygen therapy, physiotherapy, optimal pain therapy \\
\hline & $\mathrm{SvO}_{2}$ & ERAS: Protective ventilation, optimal oxygen oxygen therapy, physiotherapy, optimal pain therapy, \\
& $\mathrm{PvO}_{2}$ & ERAS: Protective ventilation, optimal oxygen therapy, physiotherapy, optimal pain therapy \\
\hline
\end{tabular}


Considering enhanced recovery after surgery (ERAS) point of view $[23,29-36]$ The objectives of ERAS is to reduce the perioperative stress which can have adverse consequences on postoperative recovery [23,29,30]. ERAS englobes measures which aim to reduce perioperative stress. These measures include optimal pain therapy, minimal invasive techniques whenever possible, optimal prevention and treatment of postoperative nausea and vomiting, prevention of postoperative organ dysfunction including infections, reducing fastening periods, favoring oral intake whenever possible, favoring early mobilization, respiratory physiotherapy to prevent pulmonary dysfunction which can lead to hypoxemia and hypoxia. Increasing oxygen saturation with oxygen therapy can increase $\mathrm{DO}_{2}, \mathrm{CaO}_{2}$ and decreases oxygen extraction ratio [33,35,36]. Oxygen consumption can be increased with the above mentioned stress situations. Reducing these situations and favoring states where $\mathrm{DO}_{2}$ is optimal is mandatory to avoid the $\mathrm{DO}_{2}$ dependency state which can have adverse effects on postoperative recovery [23].

\section{Conclusion}

GDFHT, TGDP and ERAS share the same goals. These are optimization of $\mathrm{DO}_{2}, \mathrm{VO}_{2}$ and $\mathrm{O}_{2} \mathrm{ER}$ to prevent morbidity due to organ dysfunction. These goal directed therapies are related via the $\mathrm{VO}_{2}-\mathrm{DO}_{2}$ interaction. To reach ERAS, GDFHT and TGDP are necessary. The aimed outcome of GDFHT, TGDP and ERAS is the prevention of postoperative organ dysfunction and the reduction of postoperative length of hospital stay and thus the improvement of outcome. Determinants of $\mathrm{DO}_{2}, \mathrm{VO}_{2}$, oxygen extraction and perfusion pressure underly the physiology of goal directed therapies in children. Understanding these determinants are important for optimal patient management.

\section{References}

\section{1. http://www.theses.fr/s232762}

2. Kumba C, Cresci F, Picard C, Thiry C, Albinni S, et al. (2017) Transfusion and morbi mortality factors: An observational descriptive retrospective pediatric cohort study. J Anesth Crit Care Open Access 8(4): 00315.

3. Kumba C, Taright H, Terzi E, Telion C, Beccaria K, et al. (2018) Blood product transfusion and postoperative outcome in pediatric neurosurgical patients. Curr Pediatr Res 23: 21.

4. Kumba C, Lenoire A, Cairet P, Dedieu E, Belloni I, et al. (2018) Is transfusion an independent risk factor of postoperative outcome in pediatric orthopedic surgical patients? A retrospective study. Curr Pediatr Res 23: 13.

5. Kumba C, Querciagrossa S, Blanc Thomas, Treluyer JM (2018) Transfusion and postoperative outcome in pediatric abdominal surgery. J Clin Res Anesthesiol 1(1): 1-8.

6. Kumba C (2019) A retrospective descriptive cohort study of preoperative, intraoperative and postoperative management of children in scoliosis surgery. Curr Pediatr Res 23: 23.

7. Kumba C (2019) Do goal directed therapies improve postoperative outcome in children? (Perioperative goal directed fluid and hemodynamic therapy; transfusion goal directed therapy using viscoelastic methods and enhanced recovery after surgery and postoperative out-come): a study research protocol. Acta Scientific Paediatrics 2(7): 17-19.

8. Kumba C, Melot C (2019) The era of goal directed therapies in paediatric anaesthesia and critical care. EC Emergency Medicine and Critical Care 3(5): 306-309.
9. Kumba C, Raisky O, Bonnet D, Treluyer JM (2019) Perioperative echocardiographic hemodynamic parameters and postoperative outcome in pediatric congenital heart disease: A descriptive observational prospective pilot study protocol. Int J Pediatr Neonat Care 5: 160.

10. Kumba C, Raisky O, Bonnet D, Treluyer JM (2019) Perioperative goal directed fluid and hemodynamic therapy with echocardiography in pediatric congenital heart disease: A study protocol. EC Paediatrics 8(12): 1-6.

11. Kumba C, Treluyer JM (2019) Perioperative echocardiographic hemodynamic parameters and postoperative outcome in pediatric surgical patients: A descriptive observational prospective pilot study protocol. Res Pediatr Neonatol 4(1): 294-298.

12. Kumba C, Tréluyer J (2020) Perioperative goal directed fluid and hemodynamic therapy with Echocardiography in children: A study protocol. Res Pediatr Neonatol 4(2): 306-310.

13. Kumba C (2020) Trans-thoracic echocardiographic aortic blood flow peak velocity variation, distance minute, aortic velocity time integral and postoperative outcome in pediatric surgical patients-An observational pilot study protocol. Open Journal of Internal Medicine 10(1): 90-95.

14. Kumba C (2020) Goal directed fluid and hemodynamic therapy and postoperative outcomes in children: Value of transthoracic echocardiographic aortic blood flow peak velocity variation: A multicentre randomized controlled trial protocol. Adv Pediatr Res 7: 1-6.

15. Kumba C (2019) Future evolution of intraoperative goal directed fluid and hemodynamic therapy in children. Adv Pediatr Res 6:1-2.

16. Kumba C (2020) Feasibility of intraoperative trans-thoracic echocardiography for goal directed fluid and hemodynamic therapy in children and postoperative outcome. J Neo Res Pedia Care 3(1): 180025.

17. Kumba C (2020) Trans-thoracic aortic peak velocity variation for goal directed fluid and hemodynamic therapy in children and postoperative outcome: A multicentric randomized controlled trial: Editorial. Acta Scientific Paediatrics 3(5): 01-02.

18. Kumba C (2020) Advances in pediatric and neonatal research: Impact of networking and collaborating. EC Paediatrics 9(4): 111-112.

19. Kumba C (2020) Innovating applications with trans-thoracic echocardiography in goal directed fluid and hemodynamic therapy in children. EC Clinical and Medical Case Reports 3(3): 1-3.

20. Kumba C, Loron G, Mons A, Marcus C, Grossenbacher F, et al. (2020) Veno venous extracorporeal membrane oxygenation (ECMO) in a child with hemoptysis and fontan circulation. Open Journal of Pediatrics 10(2): 280-287.

21. Kumba C, Willems A, Querciagrossa S, Harte C, Blanc T, et al. (2019) A systematic review and meta-analysis of intraoperative goal directed fluid and haemodynamic therapy in children and postoperative outcome. J Emerg Med Critical Care 5(1): 1-9.

22. Lemson J, Nusmeier A, Hoeven JG (2011). Advanced hemodynamic monitoring in critically III children. Pediatrics 128: 560-571.

23. Kumba C, Linden P (2008). Effects of sedative agents on metabolic demand. Ann Fr Anesth Reanim 27: 574-580.

24. Kumba C, Tréluyer JM (2020) Pediatric rotem sigma parameters in potential perioperative hemorrhagic surgery: An observational prospective pilot study protocol. Acta Scientific Paediatrics 3(1): 31-33.

25. Kumba C, Treluyer J (2020) Perspectives and evolution of intraoperative transfusion goal directed protocols with viscoelastic methods and perioperative outcomes in children. Res Pediatr Neonatol 4(2): 303-306.

26. Kumba C (2019) Iron deficiency with or without anemia and perspectives of perioperative management in children. Adv Pediatr Res 6: 1-4.

27. Kumba C, Querciagrossa S, Harte C, Willems A, De Cock A, et al. (2019) Study protocol for a systematic review and meta-analysis of goal directed 
intra-operative transfusion protocols guided by viscoelastic methods and perioperative outcomes in children. EC Anaesthesia 5(5): 115-119.

28. Kumba C, Querciagrossa S, Harte C, Willems A, De Cock A, Blanc T, et al. (2019) A systematic review and meta-analysis of goal directed intraoperative transfusion protocols guided by viscoelastic methods and perioperative outcomes in children. Int J Recent Sci Res 10(3): 3146631471.

29. Roy N, Parra MF, Brown ML, Sleeper LA, Nathan M, et al. (2019) Initial experience introducing an enhanced recovery program in congenital cardiac surgery. J Thorac Cardiovasc Surg, pp. 1-9.

30. Kumba C, Blanc T, De Cock A, Willems A, Harte C, et al. (2019) Protocol for rapid recovery pathways after surgery in children: A systematic review and meta-analysis. J Anes \& Cri Open Access 11(2): 42-44.

31. Kumba C, Blanc T, Willems A, Harte C, Querciagrossa S, et al. (2019) Rapid recovery pathways after surgery in children: A systematic review and meta-analysis. Med J Clin Trials Case Stud 3(3): 000211.
32. Kumba C (2019) Future perspectives of enhanced recovery after surgery in children. Int J Anaesth Res 2(3): 89-90.

33. Nasser B, Tageldein M, Al Mesned A, Kabbani M, (2017). Effects of blood transfusion on oxygen extraction ratio and central venous saturation in children after cardiac surgery. Ann Saudi Med 1(37): 31-37.

34. Filho IP, Spiess BD, Pittman RN, Barbee R, Ward KR (2005) Experimental analysis of critical oxygen delivery. Am J Physiol Heart Circ Physiol 288(3): H1071-H1079.

35. Schulze A, Whyte RK, Way C, Sinclair JC (1995) Effect of the Arterial oxygenation level on cardiac output, oxygen consumption in low birth weight infants receiving mechanical ventilation. J Pediatr 126(5 Pt 1): 777-784.

36. Vincent JL (1996) Determination of oxygen delivery and consumption versus cardiac index and oxygen extraction ratio. Critical Care Clinics 12(4): 995-1006 